\title{
A Robust Nonlinear Control Strategy for Unsupported Paraplegic Standing Using Functional Electrical Stimulation: Controller Synthesis and Simulation
}

\author{
Seyedeh Sakineh Ruhbakhsh' ${ }^{1}$ Hamid Reza Kobravi ${ }^{* *}$ \\ ${ }^{1}$ Research Center of Biomedical Engineering, Mashhad Branch, Islamic Azad University, Mashhad, Iran
}

\begin{abstract}
Background: Functional electrical stimulation (FES) applies electrical pulses to paralyzed muscles to restore their function. Closed-loop control of an FES system, incorporating the control strategies, promises to improve the performance of FES systems. Therefore, the purpose of this paper is to design a new control strategy applicable to restoring the upright standing in paraplegic patients through FES. The control strategies proposed in the previous works based on controlling the angular joint position and none of them focused on controlling the center of pressure (CoP) dynamics directly. Since the CoP is representative of posture balance dynamics, in this study, the adopted FES based control strategy designed to control the CoP dynamics directly.

Methods: In the proposed control strategy, two controllers determine the stimulation intensity of ankle muscles in a manner to restrict the CoP to a specific zone. According to the proposed strategy, until the CoP confined to the stable zone, an adaptive controller is active. When the CoP goes out of the stable zone, the adaptive controller is deactivated. Then, a sliding mode controller is activated instead of the adaptive controller. In this manner, not only the posture balance can be guaranteed, but also the emerged balance dynamics can be similar to the elicited balance dynamics in the healthy subjects.

Results: In this study, extended evaluations carried out through the simulation studies. According to the achieved results, the proposed control strategy is not only robust against the external disturbances, but also insensitive to the initial postural conditions.

Conclusion: The achieved results prove the acceptable performance of the proposed control strategy.

Keywords: Functional electrical stimulation; Center of pressure; Unsupported standing; Sliding mode control; Adaptive control.
\end{abstract}

\author{
*Correspondence to \\ Hamid Reza Kobravi, \\ Research Center of Biomedical \\ Engineering, Mashhad Branch, \\ Islamic Azad University, \\ Mashhad, Iran, \\ Tel: (98)-513-6620011-284, \\ Email: Hkobravi@mshdiau.ac.ir
}

Published online March 10, 2020

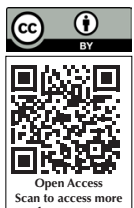

Citation: Ruhbakhsh SK, Kobravi HR. A Robust Nonlinear Control Strategy for Unsupported Paraplegic Standing Using Functional Electrical Stimulation: Controller Synthesis and Simulation. Int Clin Neurosci J. 2020;7(2):88-102. doi:10.34172/icnj.2020.07.

\section{Introduction}

The functional electrical stimulation (FES) applies electrical stimulation pulses to muscle nerves in order to produce muscle contractions. ${ }^{1}$ Also, FES is a technique that widely used to restore lost motor function. ${ }^{2,3}$ Unsupported standing using FES can enable the paraplegic patients to perform manual functions at self-selected leaning positions. ${ }^{4}$ Also, standing brings other benefits to spinal cord injury patients such as decreasing bone osteoporosis, prevention of pressure sores, prevention of orthostatic hypotension, and improvement of the digestive system..$^{5-7}$

During recent years, different control approaches, applicable to restoring the arm-free standing in paraplegia via FES, have been proposed. ${ }^{8-23}$ Masani et al ${ }^{8,9}$ introduced a feedback proportional-derivative controller to stabilize the body during quiet standing. The designed controller could facilitate stable standing even with closed-loop time delays longer than $75 \mathrm{ms.}^{8,9}$ Masani et al ${ }^{10}$ also used a proportional integral derivative (PID) controller for balance control during standing due to FES. The used PID controllers determined the stimulation intensity of the ankle plantar flexor and dorsiflexor muscle groups. ${ }^{10}$ Soetanto et $\mathrm{a}^{11}$ presented an innovative control strategy for stabilizing the standing posture of paraplegic patients. The applied control strategy based on a combination of a proportional-derivative controller and a predictive mechanism. Huryn et $\mathrm{al}^{12}$ assessed the viability and performance of an intermittent control strategy in human balance control. They showed that stability can improve by incorporating the prediction mechanism. ${ }^{12}$ Hunt et al ${ }^{13-}$

(C) 2020 The Author(s). This is an open access article distributed under the terms of the Creative Commons Attribution License (http:// creativecommons.org/licenses/by/4.0/), which permits unrestricted use, distribution, and reproduction in any medium, provided the original work is properly cited. 
${ }^{15}$ have also studied the performance of a control system with a nested-loop structure in which the outer loop was associated with regulation of the inclination angle, while the inner loop was associated with regulation of the total ankle torque. They used linear quadratic Gaussian (LQG), controllers. They also used the pole assignment method instead of LQG to achieve more extended periods of unsupported standing. ${ }^{15}$ Matjacić and Bajd ${ }^{16,17}$ proposed a new control scheme designed based upon a linear quadratic regulator optimal controller. They integrated the voluntary control of the unaffected upper limbs with the artificial control of the ankle joint movement. ${ }^{16,17}$ The Mihelj and Munih ${ }^{18}$ also addressed the integration of the voluntary trunk activity with artificial controlling the ankle torque. Audu et $\mathrm{al}^{19}$ also proposed and designed a PID feedback controller for upright posture control using FES. Kobravi and Erfanian ${ }^{20}$ proposed a new robust control framework based on the synergistic combination of an adaptive nonlinear compensator with sliding mode control. Adopting an agonist-antagonist muscle coactivation mechanism was one of the main contributions of their work..$^{20}$ Later, they utilized the proposed control approach $^{20}$ for unsupported paraplegic standing using FES. $^{21}$

In most of the mentioned works, the linear control structures associated with linear time-invariant systems have used, ${ }^{8-19}$ while the musculoskeletal system has nonlinear and time-varying properties. ${ }^{24} \mathrm{In}$ most of them, the necessity of agonist-antagonist muscle co-activation was not regarded. ${ }^{13-15}$ Also, no previous works focused on controlling the center of pressure (CoP) dynamics directly, while in the healthy subjects, the central nervous system controls the CoP directly to achieve a stable balance. Thus, this study aimed to design and evaluate a control strategy for controlling the unsupported paraplegic standing through controlling the CoP dynamics. ${ }^{25}$ In other words, the goal of the designed control mechanism was realizing a dynamic balance rather than pushing the inclination angle toward a desired fixed position. In this manner, dynamic stability, instead of static stability, will be obtained. ${ }^{26}$

\section{Materials and Methods}

The Model of Virtual Patient

In this study, a musculoskeletal model used as a virtual patient. This virtual patient model includes the skeletal part and the muscular parts. Each part of the model will elaborate in the following subsections.

\section{a) Skeletal Part}

The skeletal part is an inverted pendulum that rotates around the ankle. This part has shown in Figure $1 .^{27}$

The dynamical equation for the inverted pendulum is given by (1),

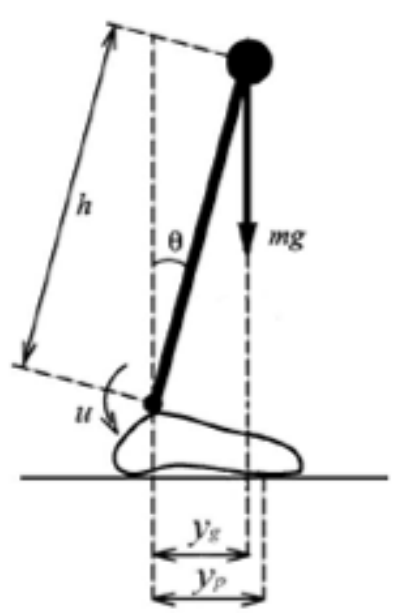

Figure 1. Single joint inverted pendulum model in which $h$ is distance of CoM from the ankle position, $\theta$ is sway angle, $u$ is total ankle torque, $M$ is the body segment mass, $g$ is acceleration of gravity, $y_{g}$ horizontal displacement of CoM, and $y_{p}$ horizontal displacement of COP. 27

$$
I_{0} \frac{d^{2} \theta}{d t}=M g h \sin (\theta)+u(\tau)+\varepsilon(\tau)
$$

Where $\theta$ is the inclination angle, $M$ is the body segment mass, $I_{0}$ is the moment of inertia, $h$ is the distance of CoM from the ankle position, $g$ is the acceleration of gravity, $u(\tau)$ is ankle torque, and $\varepsilon(\tau)$ is external disturbance torque. The set of parameters (i.e. $M, I_{0}, h$ and $g$ ) for the skeletal model have taken from ${ }^{27}$. In an inverted pendulum model of standing, the difference between the CoP and CoM is proportional to the horizontal acceleration of $\mathrm{CoM}$ in both the sagittal (anterior/posterior direction, $\mathrm{A} / \mathrm{P}$ ) and frontal (medial/ lateral direction, $\mathrm{M} / \mathrm{L}$ ) planes. Accordingly, if the horizontal acceleration of CoM is small, the CoP and CoM can be comparable and approximately equal. So, it can be supposed that horizontal displacement of $\operatorname{CoM}\left(y_{g}\right)$ and horizontal displacement of $\operatorname{CoP}\left(y_{p}\right)$ are equal. ${ }^{28}$ It means that if $y_{g}=h \sin (\theta), y_{p}$ can be defined as $y_{p}=h \sin (\theta)$

Accordingly, $\mathrm{CoP}_{\mathrm{y}}$ and the $\mathrm{CoP}_{\mathrm{y}}$ will be defined by (2) and (3), respectively.

$$
\begin{aligned}
& C o P_{y}=h \sin (\theta) \\
& \operatorname{CoP}_{y}=h \cos (\theta)
\end{aligned}
$$

Where $h$ is the position of CoM, and $\theta$ is the deviation angle. In reality, the CoP can estimate using force sensors data located on the shoe's insole.

by defining $\mathrm{x}_{1=} \theta, \mathrm{x}_{2}=\theta, \mathrm{x}_{3=} \ddot{\theta}$ using Euler scheme and substituting into (1), yields: 


$$
\begin{aligned}
& x_{1}(k+1)=x_{1}(k)+T x_{2}(k) \\
& x_{2}(k+1)=x_{2}(k)+T\left[\left(\frac{M g h}{I_{0}}\right) \sin \left(x_{1}(k)\right)+\frac{1}{I_{0}}(u(\tau)+\varepsilon(\tau))\right]
\end{aligned}
$$

During the quiet standing, the ankle strategy has applied to preserve the balance against the small external perturbation. Since this study focused on the preliminary evaluation of the proposed control strategy, the simulation studies and analyses carried out under the condition that the ankle strategy alone has applied. In this context, the hip joints and knee joints fixed in the fullextended position, and the balance preserved through activation and deactivation of the ankle extensor/flexor muscles. Accordingly, adopting the inverted pendulum rotating around the ankle joint, as the skeletal part of the musculoskeletal model, can be accountable.

\section{b) Muscular Part}

The muscular part of the virtual model consists of two muscle models with a different set of parameters. Figure 2 shows a schematic representation of the muscle model used in this study. Each muscle model includes muscle activation, muscle contraction, and segmental dynamics. ${ }^{29}$

The recruitment curve modeled using a piecewise linear recruitment function. ${ }^{29}$ A Gaussian function models the moment-angle relation, and the moment-angular velocity relation derived from a linear approximation of the forcevelocity relation described in. ${ }^{29}$ The output of the muscle contraction block is the active muscle moment $\left(M_{\text {act }}\right)$ computed by equation (5).

$$
M_{a c t}=A c t \cdot M_{m a} \cdot M_{m v}
$$

Where $M_{m a}$ is the output of the moment-angle block, and $M_{m v}$ is the output of the moment-velocity block. At last, the total external moment in equation (6) acting on the joint has computed by summing the active $\left(M_{a c t}\right)$, gravitational $\left(M_{\text {grav }}\right)$, and passive moments (viscous and elasticity moments). A linear damping function models the joint viscous moment $\left(M_{\text {viscous }}\right)$ while joint elasticity moment $\left(M_{\text {elastic }}\right)$ is modeled by a double exponential function as proposed in. ${ }^{29}$

$$
M_{\text {total }}=M_{\text {elastic }}+M_{\text {viscous }}+M_{\text {grav }}+M_{\text {act }}
$$

In order to have an uncertain model, we added a random term with uniform distribution to the intended parameters (some parameters of the skeletal part and some parameters of the muscular parts), which has the variance equal to the half of the real value of the parameter.

\section{The Proposed Cooperative Control Strategy}

The proposed strategy is a cooperative based control approach. The CoP lonely can consider as stability criteria. ${ }^{27-31}$ For the sake of obtaining dynamic stability, a stable zone has determined so that confiding the CoP signal to this zone means eliciting a desired dynamic postural control. In other words, the proposed cooperative

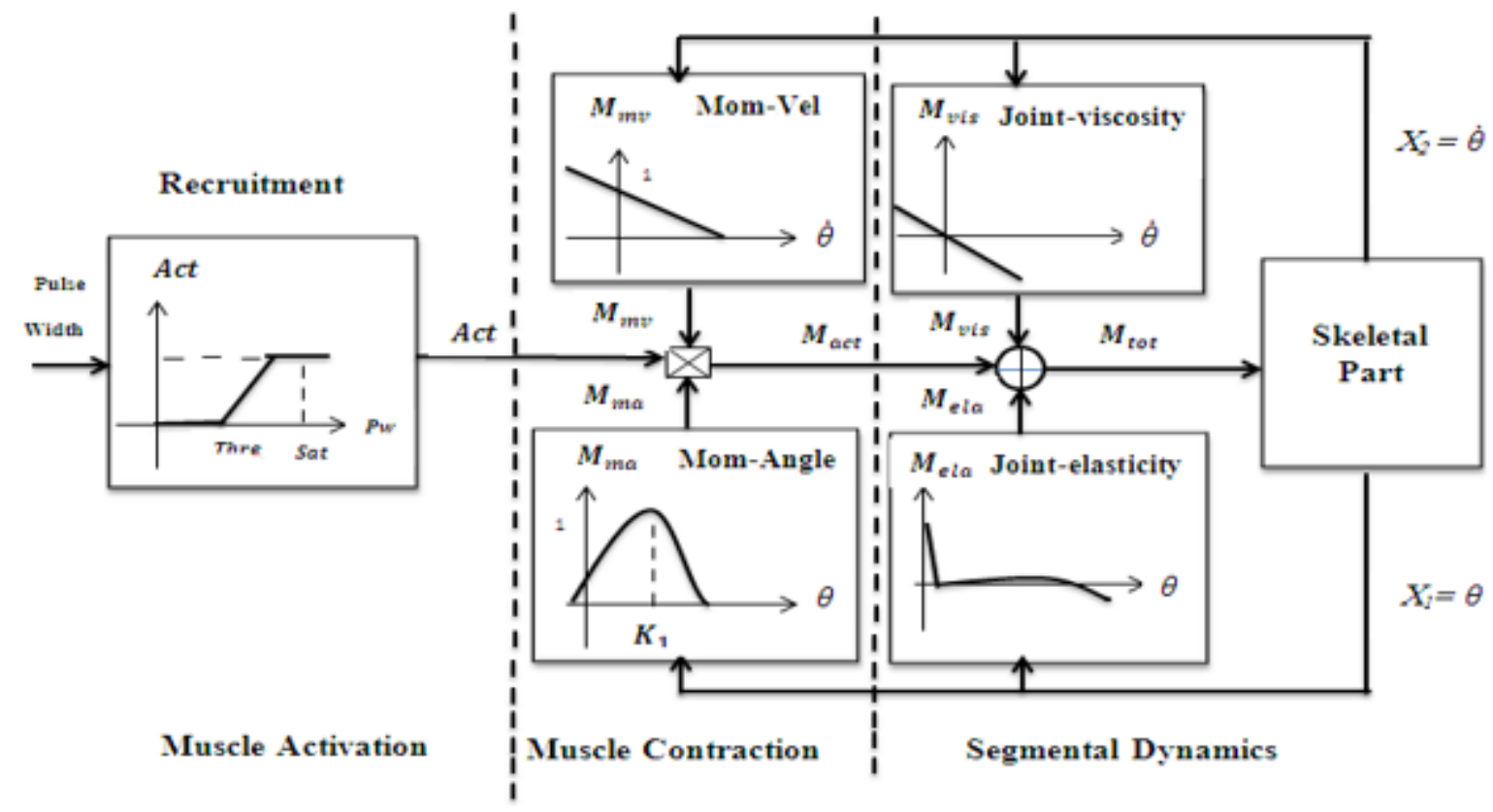

Figure 2. A Schematic Representation of the Muscle Model.29 The model describes the muscle activation properties using the muscle recruitment curve. The muscle contraction properties (moment-angle and moment-velocity), joint mechanical properties (joint-elasticity and joint-viscosity), and the leg dynamics have also described using this model. 
based control strategy seeks to bring back the CoP to a specific zone and then seeks to prevent the CoP from going outside the zone. In other words, convergence toward the zero moment point has not desired. Figure 3 shows the structure of the proposed control strategy.

The decentralized control strategy has applied to deal with muscle co-contraction. Two different closed loop control systems envisioned for adjusting the stimulation pattern of each ankle muscle group (Figure 3). In the designed control strategy, whenever the COP signal and $\mathrm{COP}^{\bullet}$ signal are out of a specific elliptical boundary, two sliding mode controllers are used to adjust the stimulation pattern of ankle plantar flexor and dorsiflexor muscle groups, and whenever the $\mathrm{CoP}$ signal and $\mathrm{COP}^{\bullet}$ signal are within a specific elliptical boundary, two stable adaptive controllers are applied to adjust the stimulation pattern of ankle plantar flexor and ankle dorsiflexor muscle groups. In this manner, adaptive controllers, along with two sliding mode controllers assigned for adjusting the stimulation pattern of each ankle muscle group. It is worth noting that adaptation laws of the utilized adaptive controllers derived in a manner that can only guarantee the stability of the control system not asymptotically stability of control system. Therefore, moving toward the zeromoment point, which means obtaining static stability, is not the control goal. Such behavior is in conformity with the behavior emerging during normal upright standing. ${ }^{30}$

As mentioned previously, an elliptical boundary has determine as a decision boundary concerning the instantaneous values of $\mathrm{CoP}$ and $\mathrm{COP}^{\bullet}$. The equation of the decision boundary is given by (7),

$$
\frac{C o P^{\bullet}}{a^{2}}+\frac{C o P^{2}}{b^{2}}=1
$$

Where $a$ and $b$ are horizontal and vertical diagonals of the ellipse. It has reported that during the quiet standing, the angular excursions of involved joints are less than degrees. ${ }^{27}$ By assigning the mentioned range for the inclination angle and using equations (2) and (3) the stable intervals relating to $\mathrm{COP}$ and $\mathrm{COP}^{\bullet}$ will be as follows:

$-0.0758<\operatorname{CoP} \leq 0.0758$

$-0.8666<\operatorname{CoP}^{\bullet} \leq 0.8666$

The equation (10) shows the decision making mechanism related to activating two designed controllers and adjusting the stimulation intensity of the ankle muscle groups.

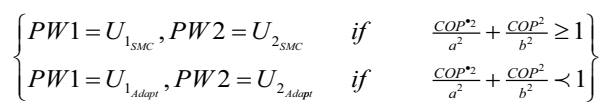

Where $U_{1_{\text {SWC }}}$ and $U_{2_{\text {SWC }}}$ are sliding mode controllers' outputs, $U_{1_{\text {Adapt }}}$ and $U_{2_{\text {Adapt }}}$ are adaptive controllers' outputs. PW1 is the pulse width value of the delivered electrical pulse to the first muscle group (ankle dorsiflexor muscle), and PW2 is the pulse width value of the delivered electrical pulse to the second muscle group (ankle plantar flexor muscle).

The designed controllers will further elaborate in the next subsections.

\section{Discrete Sliding Mode Control}

Sliding mode control (SMC) has efficiently applied for controlling the various linear and nonlinear dynamical systems. ${ }^{32}$ Since the musculoskeletal system has nonlinear dynamics, a nonlinear controller has to be utilized..$^{33}$ The sliding mode controllers are less sensitive

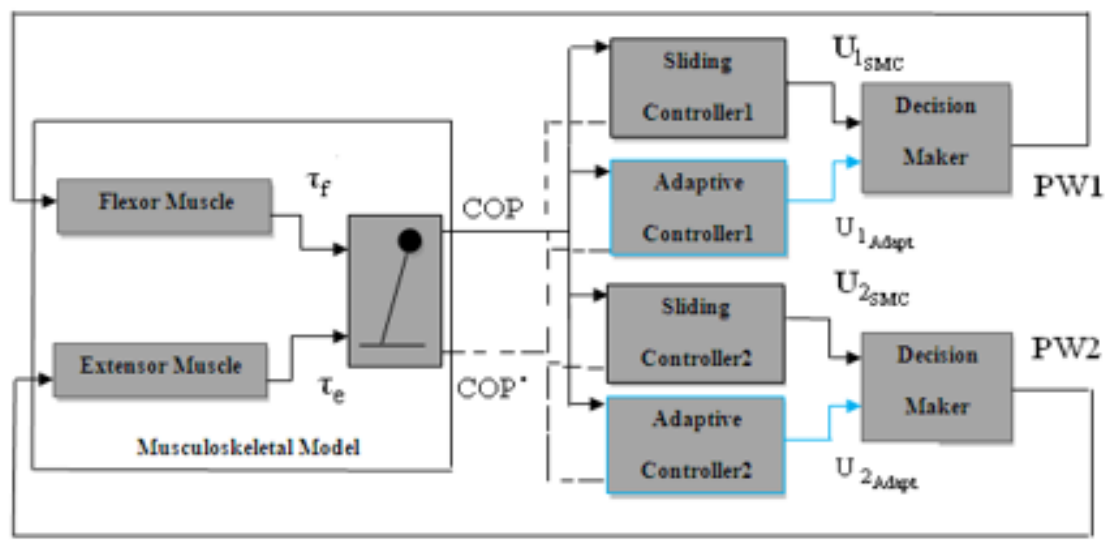

Figure 3. The Schematic of the Structure of the Proposed Control Strategy. According to a decision-making mechanism, at each time instance, one of the adopted controllers (SMC or adaptive controller) is selected intermittently for the determination of muscle stimulation pulse width. The adaptive controller selected whenever the CoP signal and its time derivative value lie within a specific zone. Otherwise, the SMC will be activated. 
concerning external disturbances and parametric uncertainties; therefore, they are appropriate for many real applications. ${ }^{34}$ The design of SMC has two parts. The first part is the design of a sliding surface as a different guide the trajectories toward the origin. The second part is designing an appropriate control law. ${ }^{35}$ The main feature of SMC is pushing the states of the system from the initial states toward a predefined sliding surface using a switching control law. ${ }^{36}$

The musculoskeletal model used in this study consists of two muscle groups and a skeletal part. If each muscle-joint system has considered a subsystem, the musculoskeletal model is a coupled system. In this study, a unique sliding mode prediction model (SMPM) for a discrete-time nonlinear uncertain coupled system has presented. The approach introduced in, ${ }^{37}$ has been utilized here.

Consider the nonlinear system containing two coupled subsystems as follows ${ }^{37}$ :

$$
\left\{\begin{array}{l}
x_{1}(k+1)=x_{2}(k) \\
x_{2}(k+1)=f_{1}(x(k))+g_{1}\left(x(k) u_{1}(k)+d_{1}(k)\right. \\
x_{3}(k+1)=x_{4}(k) \\
x_{4}(k+1)=f_{2}(x(k))+g_{2}\left(x(k) u_{2}(k)+d_{2}(k)\right.
\end{array}\right.
$$

Where $x(k)=\left[x_{1}(k) x_{2}(k) x_{3}(k) x_{4}(k)\right] \in \mathfrak{R}^{n} \quad$ is the state vector, $u_{1}(k)$ and $u_{2}(k)$ are the control signals deliver to two subsystems, $x_{1}(k)$ and $x_{2}(k)$ are the state variables of the first subsystems, $x_{3}(k)$ and $x_{4}(k)$ are the state variables of the second subsystems, $f_{1}(k), f_{2}(k), g_{1}(k)$ and $g_{2}(k)$ are nonlinear discrete functions, and $d_{1}(k)$, $d_{2}(k)$ represent dynamic coupling, parameter uncertainty, unmolded dynamics, and external disturbances.

To implement the control strategy, at first, a special SMPM is created as follows ${ }^{37}$ :

$s_{i}(k)=x_{2 i}(k)+\sigma_{i} x_{2 i-1}(k), i=1,2$

Where the $s_{i}(k)$ is sliding variable, $x_{2 i}(k)$ and $x_{2 i-1}(k)$ are state variables of subsystems, and $\sigma_{i}$ is a constant.

In the next step by applying feedback correction and receding horizon optimization, sliding mode control law is obtained as follows ${ }^{37}$ :

$$
u_{i}(k)=-\frac{h_{i}(k) g_{i}(x(k))}{g_{i}(x(k))^{2}+\lambda_{i}}
$$

Where $\lambda_{i}$ is a tunable parameter, and $h_{i}(k)$ is a nonlinear discrete function which is derived as follows, $g_{i}(x(k))$ is a nonlinear discrete function describing the uncertain dynamic system ${ }^{37}$ :

$$
s_{m_{i}}(k+1)=f_{i}(x(k))+g_{i}(x(k)) u_{i}(k)+\sigma_{i} x_{2 i}(k)+\gamma_{i} s_{i}(k)
$$

$$
\begin{aligned}
& \bar{s}_{i}(k)=S_{i}(k)-S_{m_{i}}(k) \\
& h_{i}(k)=f_{i}(x(k))+\sigma_{i} x_{2 i}(k)+\gamma_{i} s_{i}(k)+\xi_{i} \bar{s}_{i}(k)
\end{aligned}
$$

Where $f_{i}(x(k))$ is a nonlinear discrete function describing the uncertain dynamic system, $x_{2 i}(k)$ is discrete state variable, $s_{i}(k)$ is sliding variable. Also, $s_{m i}(k)$ is computed using the available system information at each time instance. Also, $\sigma_{i}$ is a constant which its value can significantly influence on the stability and dynamic performance of the designed sliding mode controller. Also, $\gamma_{i}$ is designable parameter satisfying $0 \prec \gamma_{i} \leq 1$, $\xi_{i} \in R$ is the coefficient of the feedback correction term $\left(0 \prec \xi_{i} \leq 1\right)$.

More details about the utilized discrete sliding mode control can be found in. ${ }^{37,38}$

\section{Discrete Adaptive Control}

The design process of an adaptive controller usually contains three steps: the first step is choosing a control law, the second step is choosing an adaptation law for adjusting control parameters, and the third step is analyzing the output convergence properties of the resulting control system. ${ }^{39}$

For the system defined by (1), the adaptive feedback control law and the update law are given by (15) and (16), respectively ${ }^{40}$.

$u_{i}\left(x(k)=K_{i}(k) F_{i}(x(k), i=1,2\right.$

$K_{i}(k+1)=K_{i}(k)-q^{2} B_{0}^{T}\left(P_{i}\right)\left[x_{i}(k)-B_{0} K_{i}(k) F_{i}(x(k))\right] F_{i}^{T}(x(k)) Y_{i}, i=1,2$

Where $K_{i}(k)$ is control gain, which has been defined in appendix $\mathrm{A}$ in more details, $F_{i}(x(k))$ is a nonlinear function, $q$ is a positive constant, $x_{i}(k)$ is state variable, $B_{0}$ is a symmetric and sign definite matrix, $Y_{i}$ is a positive identity matrix, and $P_{i}$ satisfies the condition $P_{i}=A_{c_{i}}^{T} P_{i} A_{i}+R_{i}$ in which $R_{i}$ is a positive identity matrix and $A_{c_{i}}=\left[A_{0} \hat{A}\right]^{-i t}$. More details about $A_{o}$ and $A$, can be found in Fu et $\mathrm{al}^{40}$ and Rugh, ${ }^{41}$ respectively.

The stability of the used adaptive controller is guaranteed using the adaptation law (equation 16) based on Lyapunov stability theory ${ }^{40}$ that has been explained in appendix B.

\section{Results}

Extended simulation studies were carried out on a musculoskeletal model as a virtual patient. The achieved results will be elaborated in details through the subsequent sections.

\section{Effects of Initial Conditions}

Two different initial conditions disturbing the balance of the musculoskeletal model $\left(x(0)=\left[\theta, \theta^{\bullet}\right]^{T}=[+5.5,0]^{T}\right.$ and $\left.x(0)=\left[\theta, \theta^{\bullet}\right]^{T}=[-5.5,0]^{T}\right)$ were determined. 
The positive initial condition is equivalent to forward leaning and the negative initial condition is equivalent to backward leaning.

The results of using the sliding mode controllers have shown in Figure 4 and Figure 5. The results of using the adaptive controllers have shown in Figure 6 and Figure 7. The results of using the cooperative control strategy have shown in Figure 8 and Figure 9. The parameters of two sliding mode controllers selected as Table $1 .^{37,38}$ The parameters of the adaptive controllers have chosen heuristically, and according to some principles presented in the literature. ${ }^{40-42}$

while $x(0)=\left[\theta, \theta^{\bullet}\right]^{T}=[+5.5,0]^{T}$, the parameters of two adaptive controllers were selected as follows:

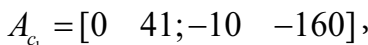

$R_{1}=0.0087 \times I_{2}$,

$Y_{1}^{c_{1}}=0.1 \times I_{4}, \quad a_{1}=102, \quad b_{1}=100.16, \quad c_{1}=-0.51$, $d_{1}=-10.2, A_{c}=\left[\begin{array}{lll}0 & 0.004 ;-3.6 & 190\end{array}\right], R_{2}=1 \times I_{2}$, $Y_{2}=90 \times I_{4}, a_{2}=10, b_{2}=9.9, c_{2}=0.1, d_{2}=1$.

While $x(0)=\left[\theta, \theta^{\bullet}\right]^{T}=[-5.5,0]^{T}$, the parameters of two adaptive controllers were selected as follows:

$$
\begin{aligned}
& A_{c_{1}}=\left[\begin{array}{lll}
0 & 1 ; 71 & 100
\end{array}\right], \quad R_{1}=0.4 \times I_{2}, \quad Y_{1}=1 \times I_{4} \\
& \text {, } a_{1}=0.001, \quad b_{1}=19, \quad c_{1}=1, \quad d_{1}=0.1 \text {, } \\
& A_{c_{2}}=\left[\begin{array}{lll}
0 & -1 ;-18.54 & 52
\end{array}\right], \quad R_{2}=28 \times I_{2}, \quad Y_{2}=8 \times I_{4} \text {, } \\
& a_{2}=3, b_{2}=1, c_{2}=2, d_{2}=1 \text {. }
\end{aligned}
$$
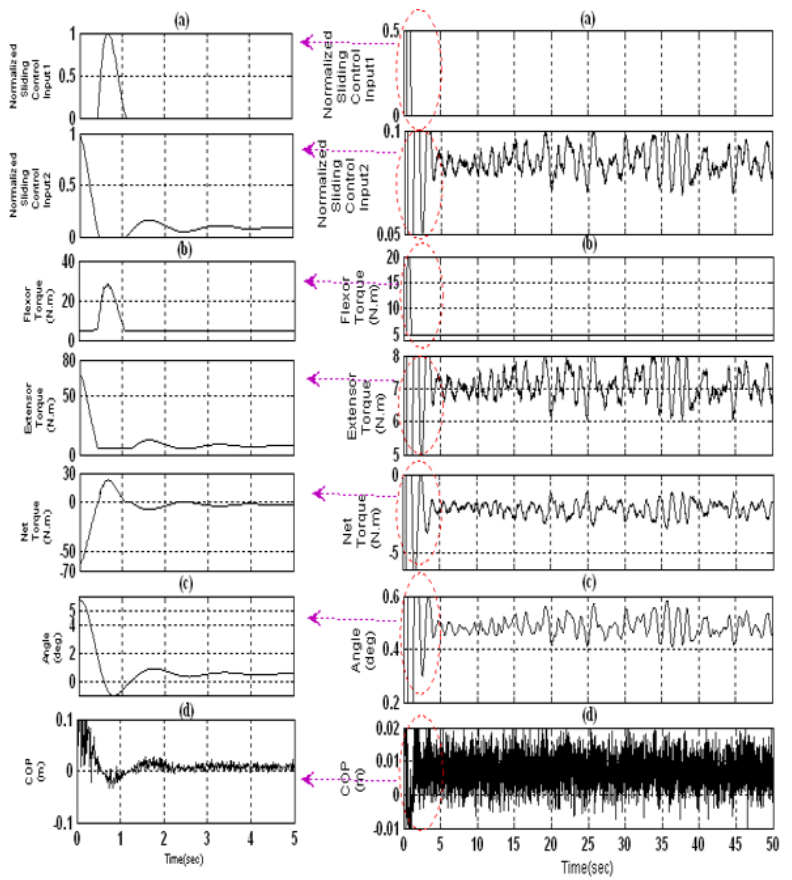

Figure 4. Simulation results of the ankle movement control obtained by the predictive SMC while $X(0)=[+5.5,0]^{\mathrm{T}}$ (a) Normalized sliding control inputs; (b) Ankle flexor, extensor, and net torques; (c) Inclination angle; and (d) Center of pressure. The left pictures are the zoomed parts of the right pictures during the time interval from 0 to 5 seconds.
Table 1. The Parameters of the Predictive Sliding Mode Controllers

\begin{tabular}{lccc}
\hline Parameter & Value & Parameter & Value \\
\hline$\sigma_{1}$ & 0.0900 & $\sigma_{2}$ & 0.000000900 \\
$\gamma_{1}$ & 0.0900 & $\gamma_{2}$ & 0.000000900 \\
$\lambda_{1}$ & -0.000100 & $\lambda_{2}$ & -0.00100 \\
$\xi_{1}$ & 0.0100 & $\xi_{2}$ & 0.0000000900 \\
\hline
\end{tabular}

Figures 4,6 , and 8 show that when the initial condition $x(0)=[+5.5,0]^{T}$, both flexor and extensor muscles contracted. However, the contraction of the extensor muscle happened sooner. Figures 5, 7, and 9 show that when the initial condition $x(0)=[-5.5,0]^{T}$, both flexor and extensor muscles contracted. Nevertheless, the contraction of the flexor muscle happened sooner.

It could see that whenever the $\mathrm{CoP}$ and $\mathrm{COP}^{\bullet}$ are out of the desired ranges (defined by the equations (8) and (9)), the sliding mode controllers generate the control signal to contract the muscle (For example the time between 0.05 $\mathrm{s}$ to $0.1 \mathrm{~s}$ in Figures 8 and 9). Also, whenever the CoP and $\mathrm{COP}^{\bullet}$ are in the desired ranges, the adaptive controllers generate the control signal to contract the muscle (For example the time $0.62 \mathrm{~s}$ to $0.65 \mathrm{~s}$ in Figures 8 and 9).

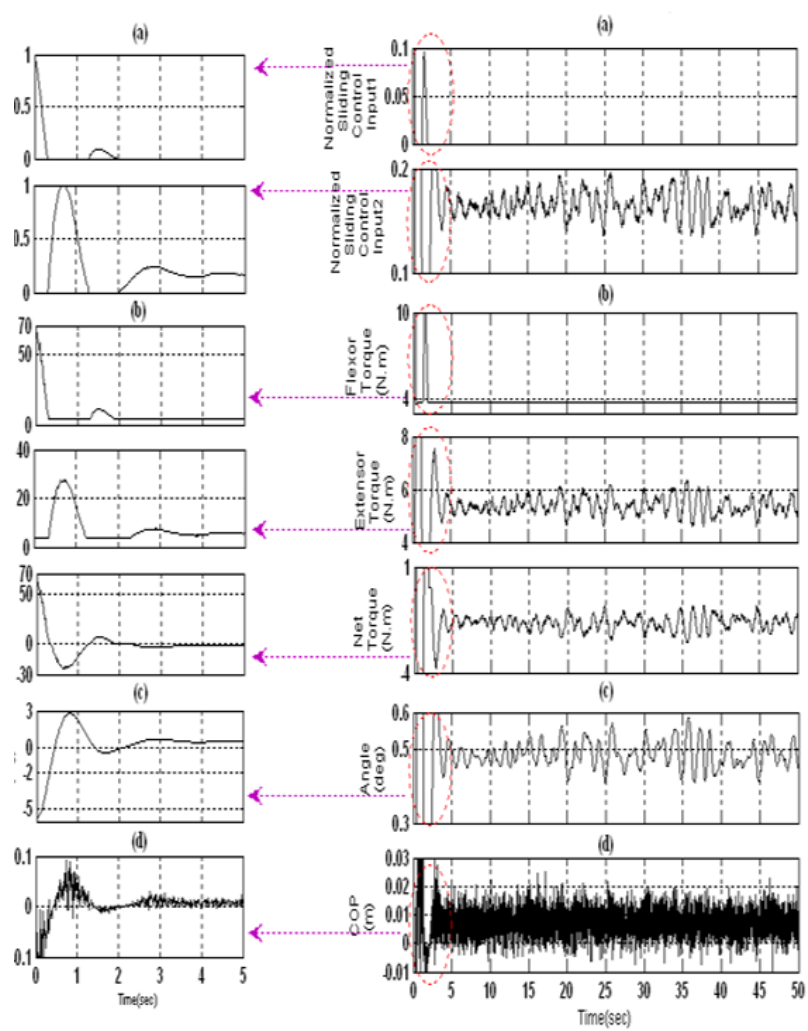

Figure 5. Simulation results of the ankle movement control obtained by the predictive SMC while $X(0)=[-5.5,0]^{\mathrm{T}}$ (a) Normalized sliding control inputs; (b) Ankle flexor, extensor, and net torques; (c) Inclination angle; and (d) Center of pressure. The left pictures are the zoomed parts of the right pictures during the time interval from 0 to 5 seconds. 

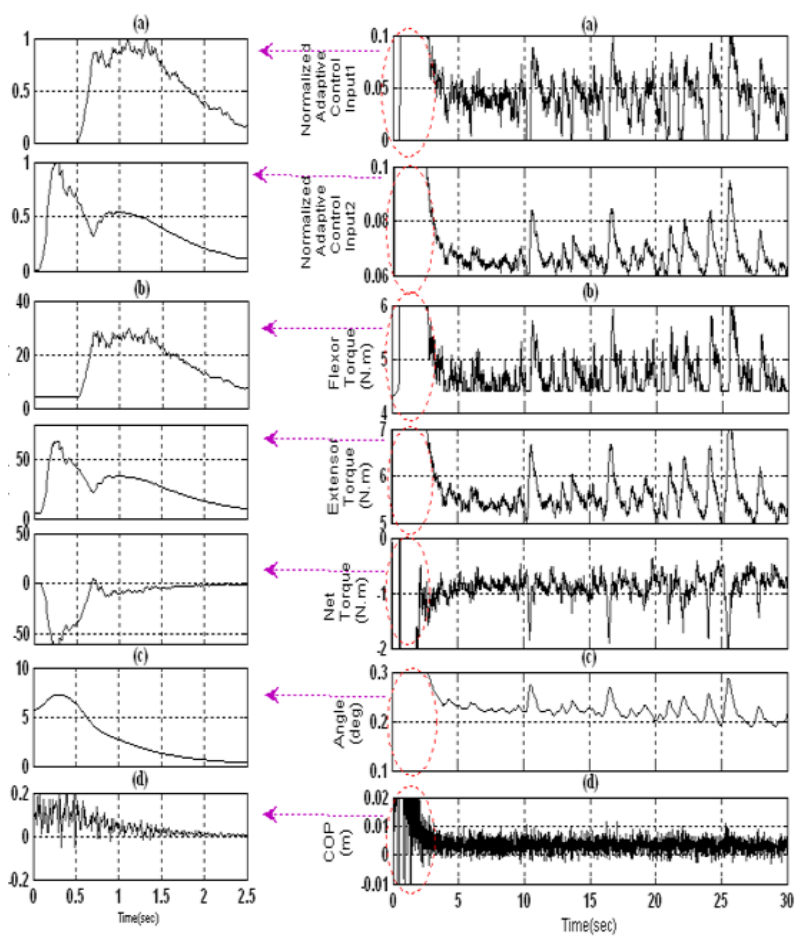

Figure 6. Simulation results of the ankle movement control obtained by the adaptive controllers while $X(0)=[+5.5,0]^{\mathrm{T}}$

(a) Normalized adaptive control inputs; (b) Ankle flexor, extensor, and net torques; (c) Inclination angle; and (d) Center of pressure. The left pictures are the zoomed parts of the right pictures during the time interval from 0 to 2.5 seconds.
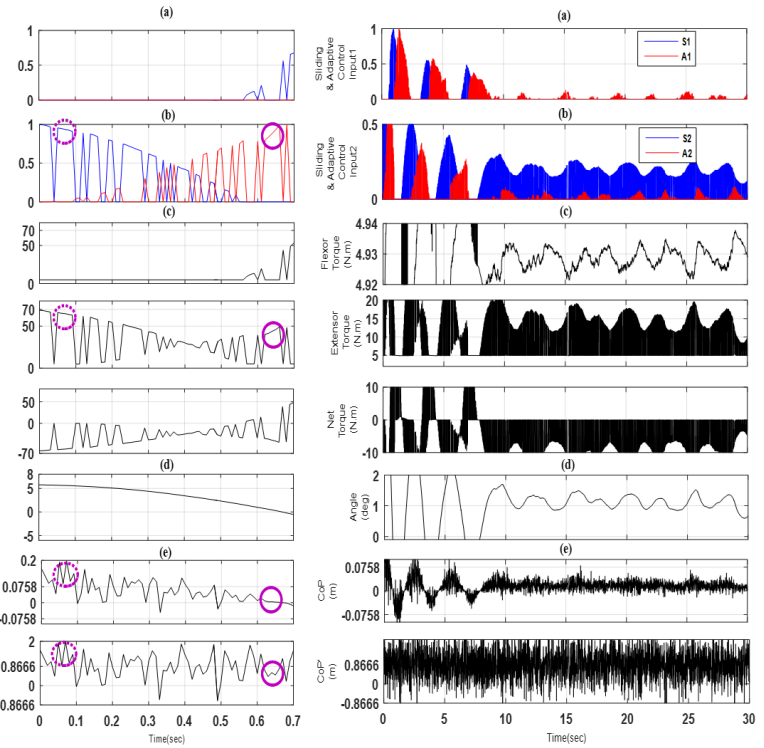

Figure 8. Simulation results of the ankle movement control obtained by the cooperative control strategy $(S M C+$ Adaptive control) while $X(0)=[+5.5,0]^{\mathrm{T}}$ (a) Normalized sliding and adaptive control input 1 which produce the ankle flexor torque; (b) Normalized sliding and adaptive control input 2 which produce the ankle extensor torque; (c) Ankle flexor, extensor, and net torques; (d) Inclination angle; and (e) Center of pressure and Velocity of center of pressure. The left pictures are the zoomed parts of the right pictures during the time interval from 0 to 0.7 seconds.
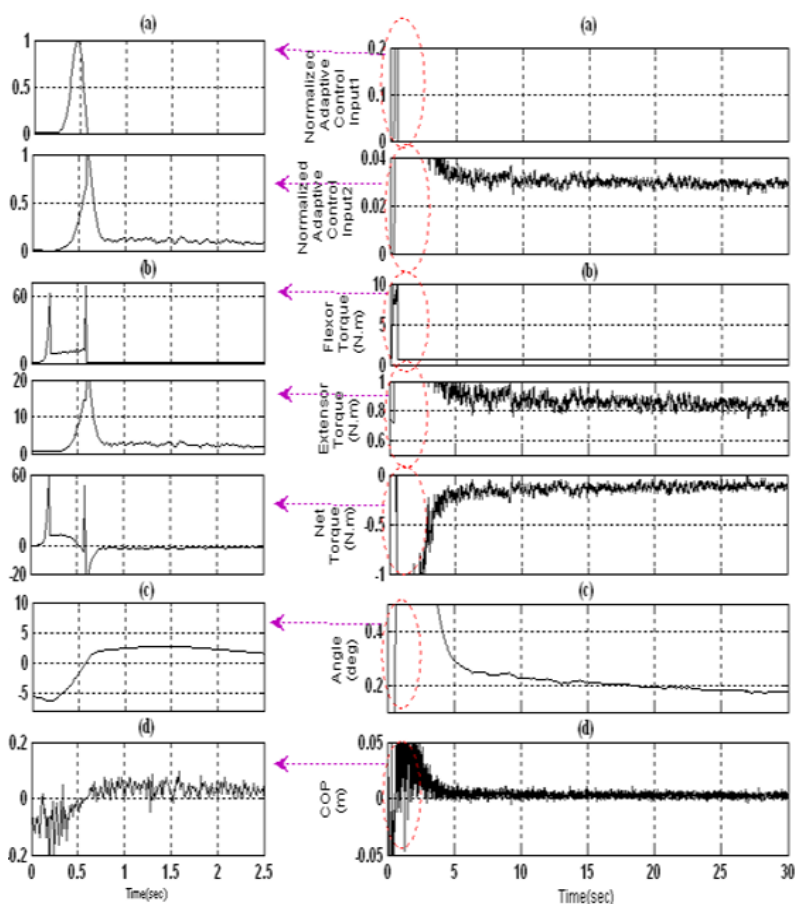

Figure 7. Simulation results of the ankle movement control obtained by the adaptive controllers while $X(0)=[+5.5,0]^{\mathrm{T}}$

(a) Normalized adaptive control inputs; (b) Ankle flexor, extensor, and net torques; (c) Inclination angle; and (d) Center of pressure. The left pictures are the zoomed parts of the right pictures during the time interval from 0 to 2.5 seconds.
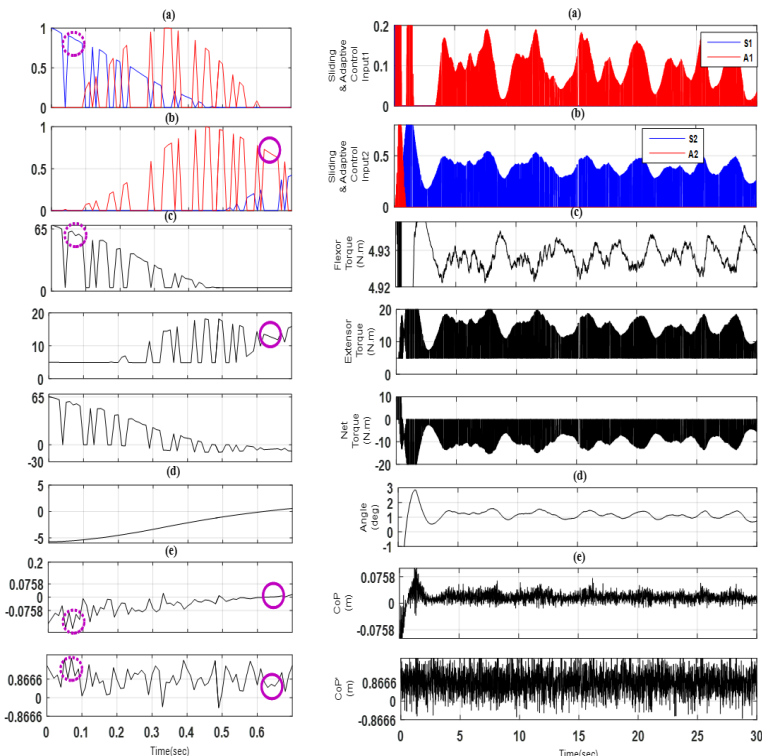

Figure 9. Simulation results of the ankle movement control obtained by the cooperative control strategy $(S M C+$ Adaptive control) while : $X(0)=[+5.5,0]^{\mathrm{T}}$ (a) Normalized sliding and adaptive control input 1 which produce the ankle flexor torque; (b) Normalized sliding and adaptive control input 2 which produce the ankle extensor torque; (c) Ankle flexor, extensor, and net torques; (d) Inclination angle; and (e) Center of pressure and Velocity of center of pressure. The left pictures are the zoomed parts of the right pictures during the time interval from 0 to 0.7 seconds. 
The intriguing point that can address concerning to the applied adaptive controller is the dependence of the controller parameters on the initial condition of the body posture. Though the parameters of the adaptive controller depend on the initial inclination angle, such dependence on the initial body position did not influence the performance of the controller. As Figures 6 and 7 show, the CoP confined to the interval $(-0.0758<$ Cop $\leq$ 0.0758 ), which regarded as the stable interval.

In this study, because of the perturbed standing situations, CoP variations may seem fast. Clearly, the stable boundary of $\operatorname{CoP}(|\operatorname{CoP}| \leq 0.0758)$ is less than the peak of $\mathrm{CoP}$ and the stable boundary of CoP velocity $(\dot{\mathrm{CoP}} \leq 0.8666)$ is near to the peak of $\mathrm{CoP}$ velocity which have reported in. ${ }^{43}$

The frequency of variation of the torque which has shown in Figures 8 and 9 is near to what has reported by Callahan et $\mathrm{al}^{44}$ some differences between real muscle and muscle model can be attributed to the inevitable unmolded dynamics of the chosen muscle model as a virtual muscle.

\section{Effects of Pulse Form External Disturbances}

In this step, two distinct pulse form external disturbances (one positive, one negative) exerted to the musculoskeletal model at the time 40 seconds after starting the control process. The time duration of each disturbance pulse

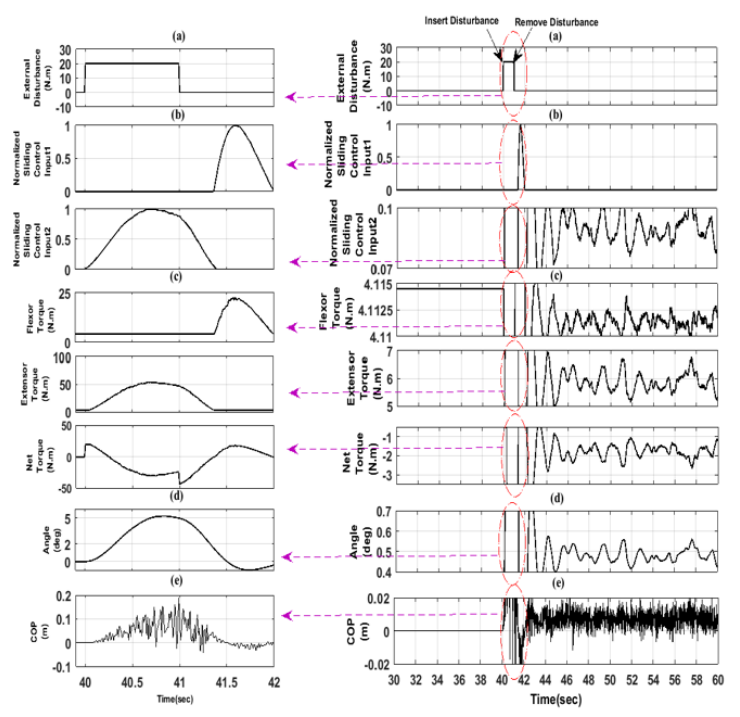

Figure 10. Simulation results of the ankle movement control obtained by the predictive SMC while the positive external disturbance is applied to the system: (a) The positive external disturbance with the amplitude of $+20 \mathrm{~N}$.m being applied to the plant during the time interval from 40 to 41 seconds; (b) Normalized sliding control inputs; (c) Ankle flexor, extensor, and net torques; (d) Inclination angle; and (e) Center of pressure. The left pictures are the zoomed parts of the right pictures during the time interval from 40 to 42 seconds. was 1 second and its amplitude was 20 N.m (which was approximately $30 \%$ of peak-to-peak generated torque during the disturbance-free trial).

The results of using the sliding mode controllers have been shown in Figure 10 and Figure 11. The results of using the adaptive controllers have been shown in Figure 12 and Figure 13. The results of using the cooperative control strategy have been shown in Figure 14 and Figure 15.

The parameters of two sliding mode controllers were selected as Table 1.

While applying the positive external pulse, the parameters of two adaptive controllers were selected as follows:

$$
\begin{aligned}
& A_{c_{1}}=\left[\begin{array}{lll}
0 & 41 ;-10 & -160
\end{array}\right], R_{1}=0.0087 \times I_{2}, \\
& Y_{1}=0.1 \times I_{4}, a_{1}=40, b_{1}=39.28, c_{1}=-0.2, d_{1}=-4, \\
& A_{c_{2}}=\left[\begin{array}{lll}
0 & 0.004 ;-3.6 & 190
\end{array}\right], R_{2}=0.0001 \times I_{2}, \\
& Y_{2}=90 \times I_{4}, a_{2}=4, b_{2}=9.82, c_{2}=0.01, d_{2}=1 .
\end{aligned}
$$

While applying the negative external pulse, the parameters of two adaptive controllers were selected as follows:

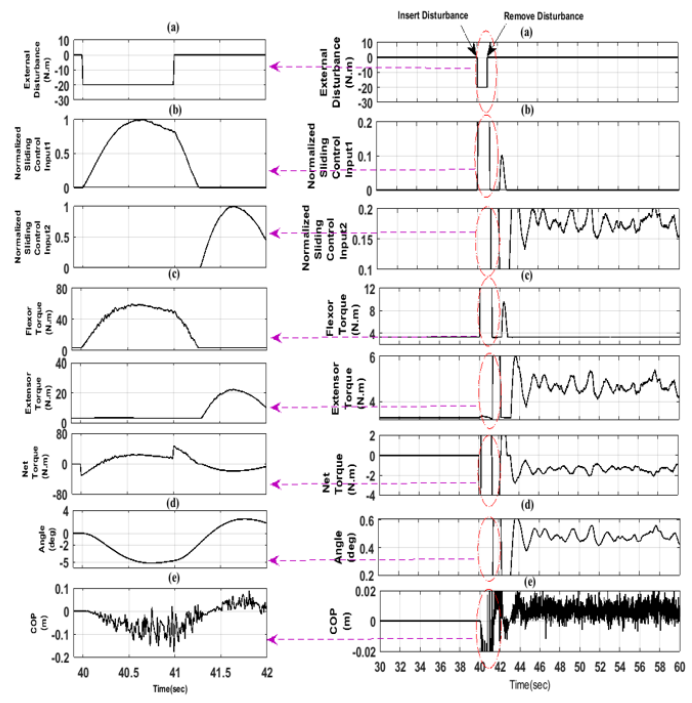

Figure 11. Simulation results of the ankle movement control obtained by the predictive SMC while the negative external disturbance is applied to the system: (a) The negative external disturbance with the amplitude of $-20 \mathrm{~N} . \mathrm{m}$ being applied to the plant during the time interval from 40 to 41 seconds; (b) Normalized sliding control inputs; (c) Ankle flexor, extensor, and net torques; (d) Inclination angle; and (e) Center of pressure. The left pictures are the zoomed parts of the right pictures during the time interval from 40 to 42 seconds. 


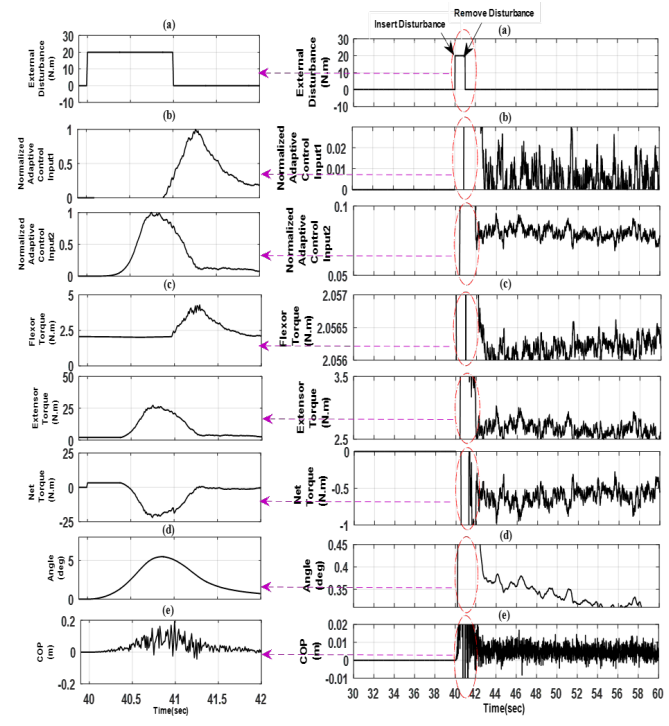

Figure 12. Simulation results of the ankle movement control obtained by the adaptive controllers while the positive external disturbance is applied to the system: (a) The positive external disturbance with the amplitude of $+20 \mathrm{~N}$.m being applied to the plant during the time interval from 40 to 41 seconds; (b) Normalized adaptive control inputs; (c) Ankle flexor, extensor, and net torques; (d) Inclination angle; and (e) Center of pressure. The left pictures are the zoomed parts of the right pictures during the time interval from 40 to 42 seconds.

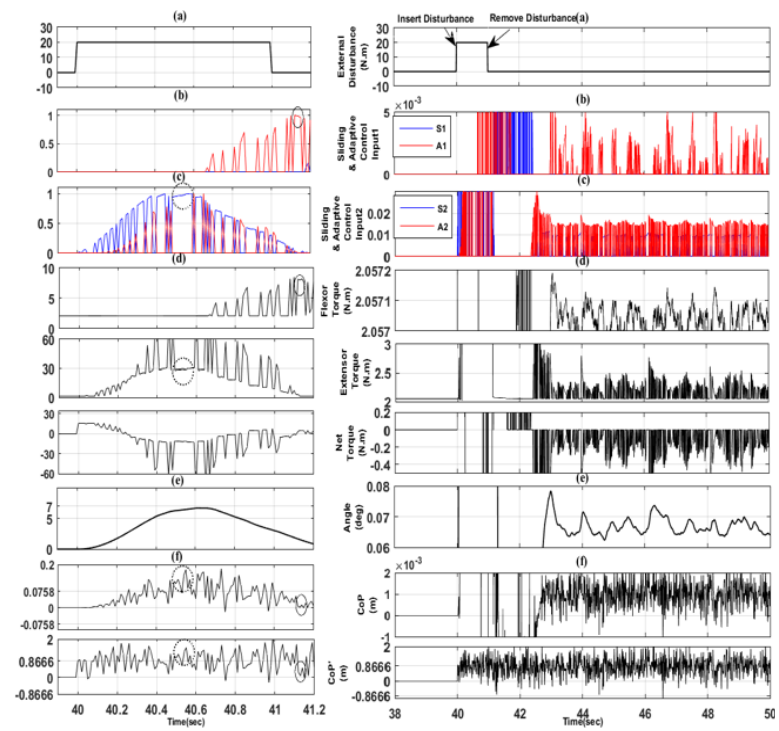

Figure 14. Simulation results of the ankle movement control obtained by the cooperative control strategy(SMC + Adaptive control) while the positive external disturbance is applied to the system: (a) The positive external disturbance with the amplitude of +20 N.m being applied to the plant during the time interval from 40 to 41 seconds (b) Normalized sliding and adaptive control input 1 which produce the ankle flexor torque; (c) Normalized sliding and adaptive control input 2 which produce the ankle extensor torque; (d) Ankle flexor, extensor, and net torques; (e) Inclination angle; and (f) Center of pressure and Velocity of center of pressure. The left pictures are the zoomed parts of the right pictures during the time interval from 40 to 41.2 seconds.

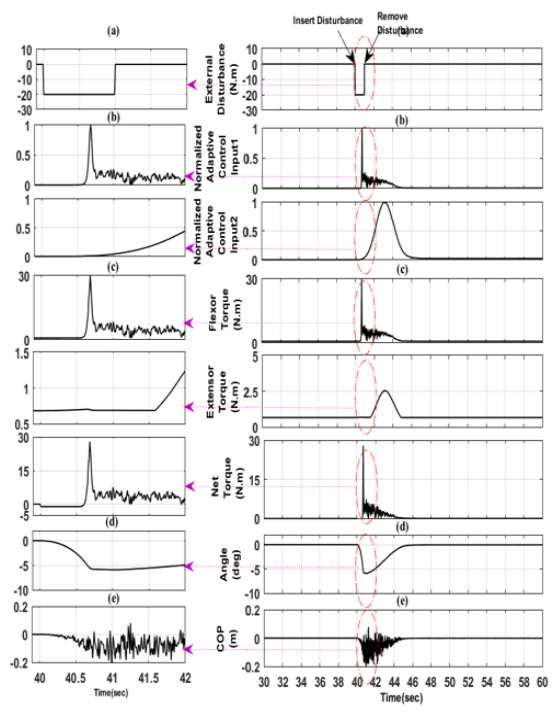

Figure 13. Simulation results of the ankle movement control obtained by the adaptive controllers while the negative external disturbance is applied to the system: (a) The positive external disturbance with the amplitude of $-20 \mathrm{~N} . \mathrm{m}$ being applied to the plant during the time interval from 40 to 41 seconds; (b) Normalized adaptive control inputs; (c) Ankle flexor, extensor, and net torques; (d) Inclination angle; and (e) Center of pressure. The left pictures are the zoomed parts of the right pictures during the time interval from 40 to 42 seconds.
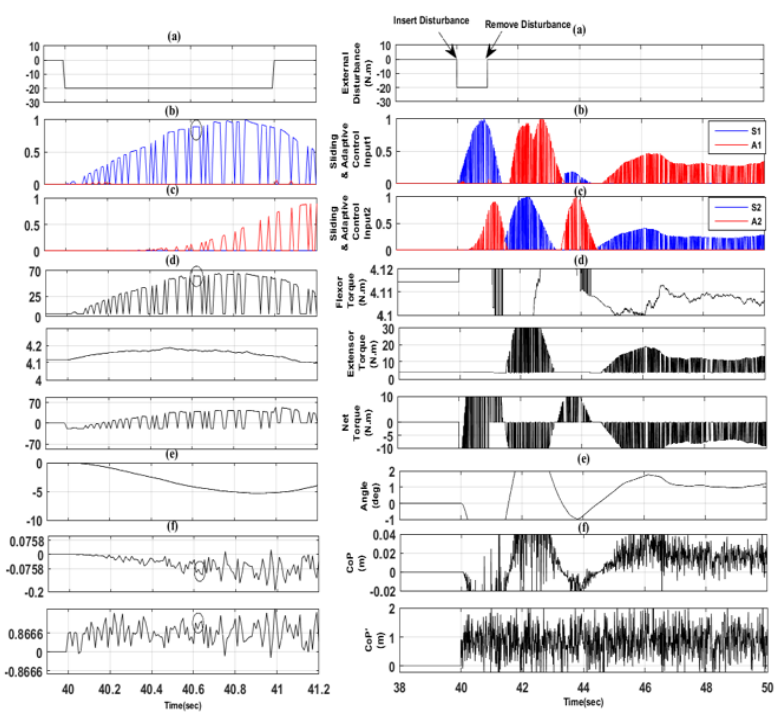

Figure 15. Simulation results of the ankle movement control obtained by the cooperative control strategy(SMC + Adaptive control) while the negative external disturbance is applied to the system: (a) The negative external disturbance with the amplitude of $-20 \mathrm{~N}$. $\mathrm{m}$ being applied to the plant during the time interval from 40 to 41 seconds (b) Normalized sliding and adaptive control input 1 which produce the ankle flexor torque; (c) Normalized sliding and adaptive control input 2 which produce the ankle extensor torque; (d) Ankle flexor, extensor, and net torques; (e) Inclination angle; and $(f)$ Center of pressure and Velocity of center of pressure. The left pictures are the zoomed parts of the right pictures during the time interval from 40 to 41.2 seconds. 
$A_{c_{1}}=\left[\begin{array}{lll}0 & 0 ;-0.1 & 1\end{array}\right], R_{1}=1 \times I_{2}, Y_{1}=-6 \times I_{4}$,

$a_{1}=2, b_{1}=11, c_{1}=100, d_{1}=-200$,

$A_{c_{2}}=\left[\begin{array}{lll}0 & -1 ;-0.8 & 0.004\end{array}\right], R_{2}=36 \times I_{2}, Y_{2}=2 \times I_{4}$,

$a_{2}=0.009, b_{2}=30, c_{2}=20, d_{2}=10$.

Figures 10, 12, and 14 show that while applying the positive pulse, both flexor and extensor muscles contracted. However, contraction of the extensor muscle happens sooner, or the value of the extensor torque was more. Also, Figures 11, 13, and 15 show that while applying the negative pulse, both flexor and extensor muscles contracted. Nevertheless, the contraction of the flexor muscle happens sooner, and the value of the flexor torque is more. It could see that whenever the $\mathrm{CoP}$ and $\mathrm{COP}^{\bullet}$ are out of the desired ranges (defined by the equations 8 and 9) sliding mode controllers generated control signal to contract the muscle (For example the time between 40.5 s to 40.6 s). Also, whenever the $\mathrm{CoP}$ and $\mathrm{COP}^{\bullet}$ are in the desired ranges, the adaptive controller generated the control signal to contract the muscle (For example: the time $41.12 \mathrm{~s}$ to $41.16 \mathrm{~s}$ ).

Also, it could see that whenever the $\mathrm{CoP}$ and $\mathrm{COP}^{\bullet}$ are

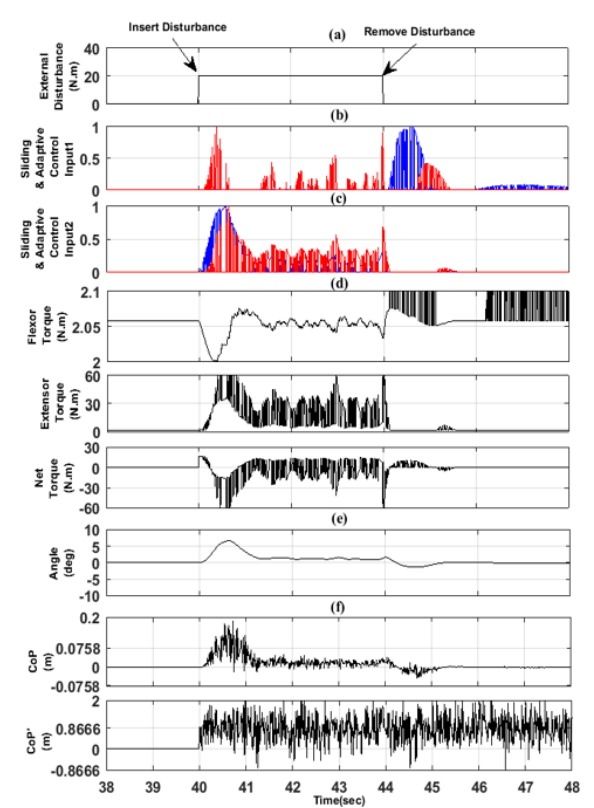

Figure 16. Simulation results of the ankle movement control obtained by the cooperative control strategy(SMC + Adaptive control) while the positive external disturbance is applied to the system:(a) The positive external disturbance with the amplitude of $+20 \mathrm{~N}$.m being applied to the plant in the time interval from 40 to 44 seconds (b) Normalized sliding and adaptive control input 1 which produce the ankle flexor torque; (c) Normalized sliding and adaptive control input 2 which produce the ankle extensor torque; (d) Ankle flexor, extensor, and net torques; (e) Inclination angle; and (f) Center of pressure and Velocity of center of pressure. out of the desired ranges (defined by the equations 8 and 9), sliding mode controller activated again and generated the control signal to contract the muscle (For example the time between $40.61 \mathrm{~s}$ to $40.65 \mathrm{~s}$ in Figure 15).

The performance of the cooperative control strategy evaluated, when two pulse form external disturbances (one positive, one negative) exerted at the time $40 \mathrm{~s}$. The time duration of disturbance pulses was $4 \mathrm{~s}$ and their amplitude was 20 N.m. Figures 16 and 17 show the results. The frequency of variation of the torque which has seen in Figures 14-17 is near to what has been reported by Callahan et al, ${ }^{44}$ some differences between real muscle and muscle model can attribute to the inevitable unmolded dynamics of the chosen muscle model as the virtual muscle.

\section{Effects of Pulse Trains Form External Disturbances}

In this step, two distinct pulse trains form external disturbances (one positive, one negative). It has exerted to the musculoskeletal model. The time duration of each disturbance pulse was $10 \mathrm{~s}$, and its amplitude was 20 N.m. The results of using the sliding mode controllers have shown in Figure 18 and Figure 19. The results of using the adaptive controllers have shown in Figure 20 and Figure

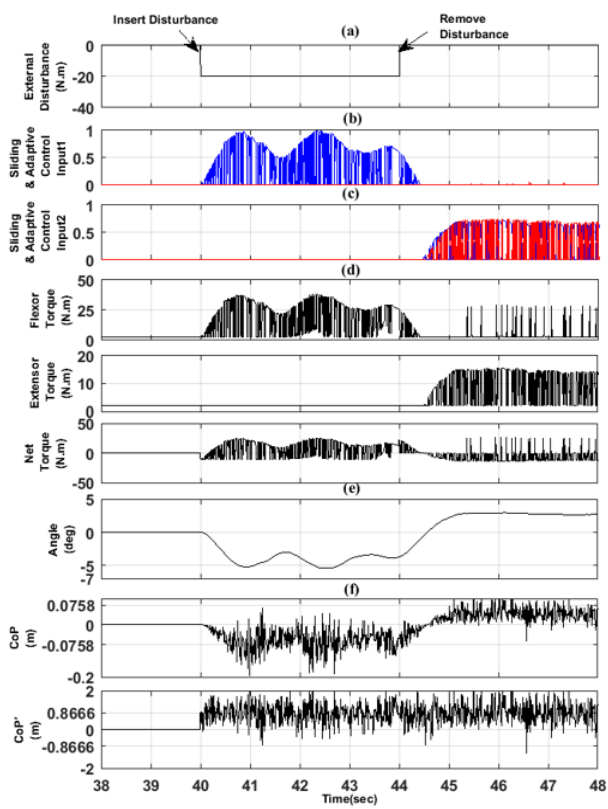

Figure 17. Simulation results of the ankle movement control obtained by the cooperative control strategy(SMC + Adaptive control) while the negative external disturbance is applied to the system: (a) The negative external disturbance with the amplitude of $-20 \mathrm{~N} . \mathrm{m}$ being applied to the plant in the time interval from 40 to 44 seconds (b) Normalized sliding and adaptive control input 1 which produce the ankle flexor torque; (c) Normalized sliding and adaptive control input 2 which produce the ankle extensor torque; (d) Ankle flexor, extensor, and net torques; (e) Inclination angle; and ( $f$ ) Center of pressure and Velocity of center of pressure. 


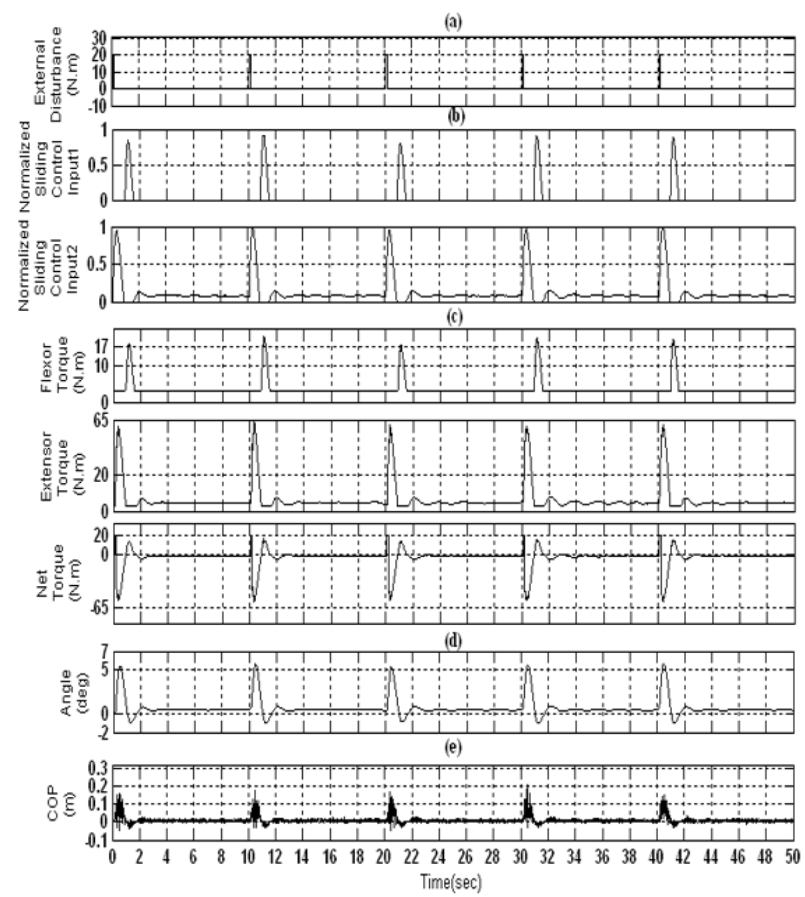

Figure 18. Simulation results of the ankle movement control obtained by the predictive SMC while the external perturbation in the form of positive pulse trains is sequentially applied to the system: (a) The positive external disturbance with the amplitude of $+20 \mathrm{~N} . \mathrm{m}$ and pulse duration equal to $10 \mathrm{~s}$; (b) Normalized sliding control inputs; (c) Ankle flexor, extensor, and net torques; (d) Inclination angle; and (e) Center of pressure.

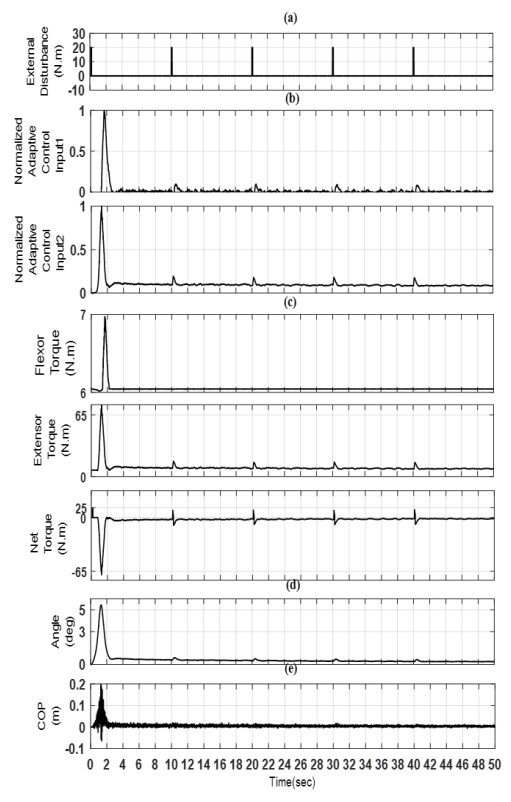

Figure 20. Simulation results of the ankle movement control obtained by the adaptive control while the external perturbation in the form of positive pulse trains is sequentially applied to the system: (a) The positive external disturbance with the amplitude of +20 N.m and pulse duration equal to $10 \mathrm{~s}$; (b) Normalized adaptive control inputs; (c) Ankle flexor, extensor, and net torques; (d) Inclination angle; and (e) Center of pressure.
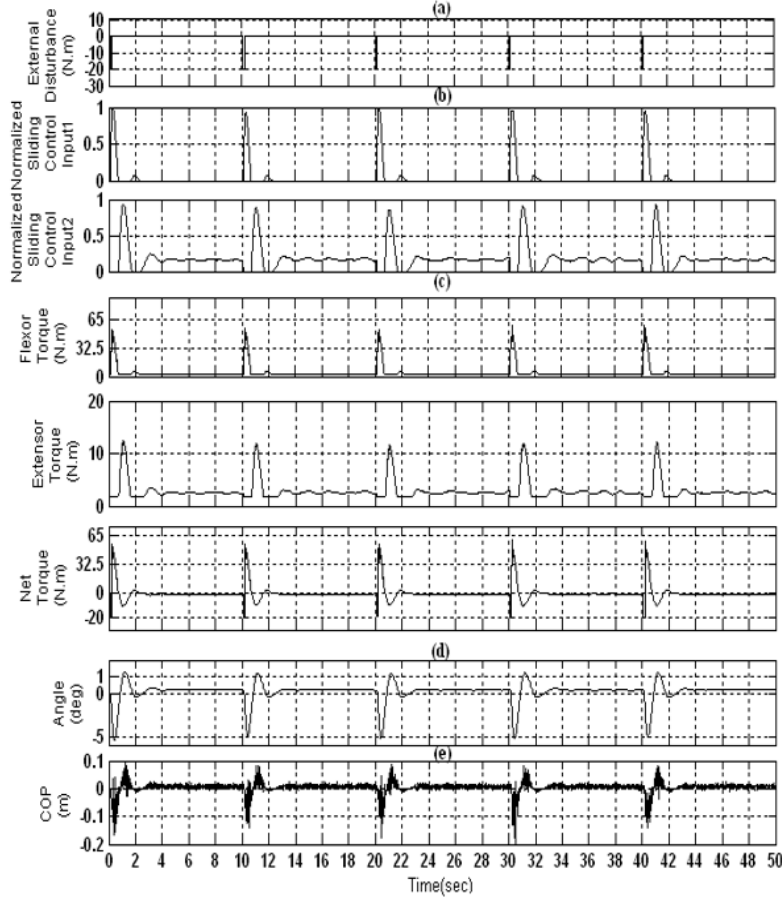

Figure 19. Simulation results of the ankle movement control obtained by the predictive SMC while the external perturbation in the form of negative pulse trains is sequentially applied to the system: (a) The negative external disturbance with the amplitude of $-20 \mathrm{~N} . \mathrm{m}$ and pulse duration equal to $10 \mathrm{~s}$; (b) Normalized sliding control inputs; (c) Ankle flexor, extensor, and net torques; (d) Inclination angle; and (e) Center of pressure.

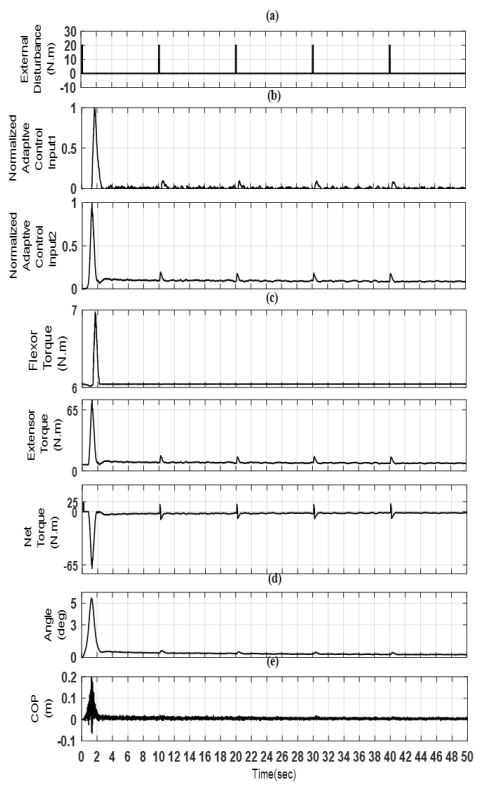

Figure 21. Simulation results of the ankle movement control obtained by the adaptive control while the external perturbation in the form of negative pulse trains is sequentially applied to the system: (a) The negative external disturbance with the amplitude of $-20 \mathrm{~N} . \mathrm{m}$ and pulse duration equal to $10 \mathrm{~s}$; (b) Normalized adaptive control inputs; (c) Ankle flexor, extensor, and net torques; (d) Inclination angle; and (e) Center of pressure 


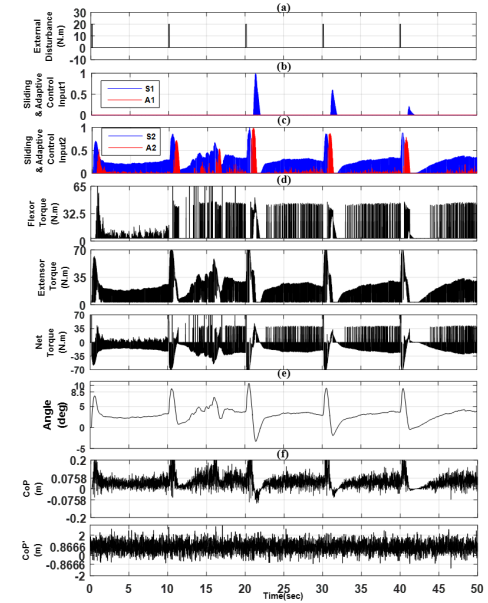

Figure 22. Simulation results of the ankle movement control obtained by the cooperative control strategy (SMC + Adaptive control) while the external perturbation in the form of positive pulse trains is sequentially applied to the system: (a) The positive external disturbance with the amplitude of +20 N.m and pulse duration equal to $10 \mathrm{~s}$; (b) Normalized sliding and adaptive control input 1 which produce the ankle flexor torque; (c) Normalized sliding and adaptive control input 2 which produce the ankle extensor torque; (d) Ankle flexor, extensor, and net torques; (e) Inclination angle; and ( $f$ ) Center of pressure and Velocity of center of pressure.

21. The results of using the cooperative control strategy have shown in Figure 22 and Figure 23.

The parameters of two sliding mode controllers selected as Table 1.

While applying the positive external pulse train, the parameters of two adaptive controllers were selected as follows:

$A_{c_{1}}=\left[\begin{array}{lll}0 & 41 ;-10 & -160\end{array}\right], R_{1}=0.0087 \times I_{2}, Y_{1}=0.1 \times I_{4}, a_{1}=40$,

$b_{1}=39.28, c_{1}=-0.2, d_{1}=-4, A_{c_{2}}=\left[\begin{array}{lll}0 & 0.004 ;-3.6 & 190\end{array}\right]$,

$R_{2}=0.0001 \times I_{2}, Y_{2}=90 \times I_{4}, a_{2}=4, b_{2}=9.82, c_{2}=0.01, d_{2}=1$.

While applying the negative external pulse train, the parameters of two adaptive controllers were selected as follows:

$A_{c_{1}}=\left[\begin{array}{lll}0 & 0 ;-0.1 & 1\end{array}\right], R_{1}=1 \times I_{2}, Y_{1}=-6 \times I_{4}, a_{1}=2, b_{1}=11$,

$c_{1}=100, d_{1}=-200, A_{c_{2}}=\left[\begin{array}{lll}0 & -1 ;-0.8 & 0.004\end{array}\right], R_{2}=36 \times I_{2}$,

$Y_{2}=2 \times I_{4}, a_{2}=0.009, b_{2}=30, c_{2}=20, d_{2}=10$.

Figures 18, 20, and 22 show that while applying the positive pulse trains, the contraction of the extensor muscle happens sooner, or the value of the extensor torque was more. Also, Figures 19, 21, and 23 show that while applying the negative pulse, the contraction of the flexor muscle happens sooner, and the value of the flexor torque is more. Also, the frequency of variation of the

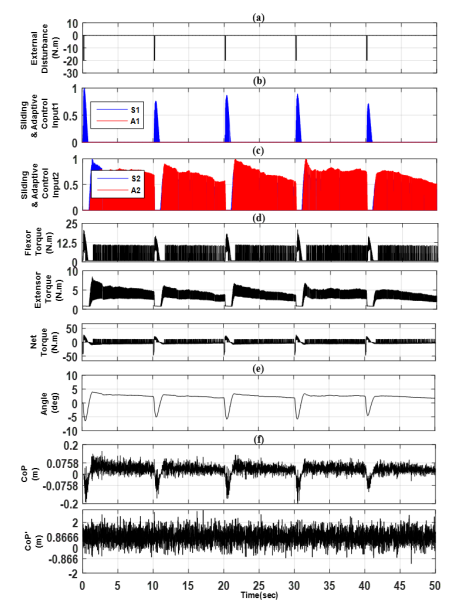

Figure 23. Simulation results of the ankle movement control obtained by the cooperative control strategy(SMC + Adaptive control) while the external perturbation in the form of negative pulse trains is sequentially applied to the system: (a) The negative external disturbance with the amplitude of -20 N.m and pulse duration equal to $10 \mathrm{~s}$; (b) Normalized sliding and adaptive control input 1 which produce the ankle flexor torque; (c) Normalized sliding and adaptive control input 2 which produce the ankle extensor torque; (d) Ankle flexor, extensor, and net torques; (e) Inclination angle; and ( $f$ ) Center of pressure and Velocity of center of pressure.

torque which has seen in Figure 22 and 23 is near to what has been reported by Callahan et $\mathrm{al}^{4}{ }^{44}$ some differences between real muscle and muscle model can attribute to the inevitable unmolded dynamics of the chosen muscle model as the virtual muscle.

\section{Quantitative Evaluations}

At last, the performance of the three used control schemes compared. For this aim, three quantitative measures, including steady state error, settling time, and the number of times that the $\mathrm{CoP}$ and $\mathrm{COP}^{\bullet}$ entered and exited from the stable zone, were computed and analyzed.

When the predictive SMC used as a lonely control strategy, the control goal was making the output error converged to zero. Therefore, the steady state error was

Table 2. The values of steady state error when the predictive SMC was used as a lonely control strategy

\begin{tabular}{lc}
\hline Different Conditions & Steady State Error (degree) \\
\hline Positive initial condition & $0.2^{\circ}$ \\
Negative initial condition & $0.2^{\circ}$ \\
Positive external pulse & $0.2^{\circ}$ \\
Negative external pulse & $0.2^{\circ}$ \\
Positive external pulse train & 0.1 \\
Negative external pulse train & $0.1^{\circ}$ \\
Average & $0.17^{\circ}$ \\
\hline
\end{tabular}


computed and analyzed only when the designed SMC used a single control strategy. Table 2 shows the computed values of steady state errors achieved when the SMC used as a lonely control strategy. The average value is less than $0.2^{\circ}$. Since the range of motion of the inclination angle can be between $-90^{\circ}$ to $90^{\circ}$, such steady state error can be acceptable.

In the second step, the settling time was computed. The settling time is the time required for the system output (inclination angle) to reach within a boundary around the zero. The computed times have been reported in Table 3. Obviously, using the cooperative control strategy conduced to observe the shortest settling time (Average value: $3.7 \mathrm{~s}$ ).

In the third stage, the number of times that the COP and $\mathrm{COP}^{\bullet}$ entered the stable zone and the number of times that the $\mathrm{COP}$ and $\mathrm{COP}^{\bullet}$ got out the stable zone were counted. Counting results have been shown in Table 4. Clearly, the most number of times which the COP and $\mathrm{COP}^{\bullet}$ were confined to the desired zone was observed when the adaptive controller was adopted. But, in terms of counting results, the performance of the adaptive controller and combined control method are comparable.

\section{Discussion}

In the previous subsections, the evaluation results of the proposed control strategy discussed from a technical point of view. Scrutinizing, in the achieved results, reveals some different aspects demonstrated in the following subparts.

\section{Robustness Concerning Time-Varying Properties}

As mentioned previously, the random numbers with uniform distribution added to some parameters of the musculoskeletal model. In this manner, the plant became a non-deterministic and time-varying system. The acceptable performance of the controller in such context proves the robustness of the control system concerning the time-varying properties of the musculoskeletal system. One of the notorious events that give rise to time-varying behavior is muscle fatigue. FES expedites the occurrence of muscle fatigue. Although the used muscle model does not simulate the occurrence of muscle fatigue, according to the results, it can expect that this controller can cope with muscle fatigue.

\section{Imitating the Normal Standing Dynamics}

In all the previous similar works, the control strategies mostly designed in a manner that pushes the inclination angle toward a set point to preserve the posture balance during the quiet standing. However, in this manner, the controller imposes a closed-loop dynamic that mimics

Table 3. The values of the settling time computed as the different control schemes were used in presence of external disturbances

\begin{tabular}{|c|c|c|c|}
\hline \multirow{2}{*}{ Different Conditions } & SMC & Adaptive Control & $\begin{array}{c}\text { Cooperative Control Strategy } \\
\text { (Adaptive \& SMC) }\end{array}$ \\
\hline & Settling Time (s) & Settling Time (s) & Settling Time (s) \\
\hline Positive initial condition & 4 & 4 & 7 \\
\hline Negative initial condition & 4 & 4.75 & 3 \\
\hline Positive external pulse & 5 & 3 & 2.5 \\
\hline Negative external pulse & 5 & 5 & 5 \\
\hline Positive external pulse train & 2.5 & 3 & 2.7 \\
\hline Negative external pulse train & 2.5 & 4 & 2.5 \\
\hline Average & 3.83 & 3.96 & 3.7 \\
\hline
\end{tabular}

Table 4. the number of times that the COP and $\mathrm{COP}^{\bullet}$ were inside and outside of the ellipse (in terms of percentage)

\begin{tabular}{lccc}
\hline \multirow{2}{*}{ Different conditions } & Adaptive Control & \multicolumn{1}{c}{ Adaptive Control } & \multicolumn{2}{c}{ Combined Control Structure } & Combined Control Structure \\
\cline { 2 - 4 } & In the Ellipse & Out of the Ellipse & In the Ellipse \\
\hline Positive initial condition & 49.88 & 50.12 & 48.13 \\
Negative initial condition & 50.04 & 49.96 & 48.43 \\
Positive external pulse & 49.88 & 50.12 & 49.92 \\
Negative external pulse & 49.60 & 50.40 & 48.85 \\
Positive external pulse train & 49.86 & 50.14 & 40.05 \\
Negative external pulse train & 49.75 & 50.25 & 43.59 \\
Average & $\rightarrow 49.83$ & 50.17 & 51.15 \\
\hline
\end{tabular}


the behavior which differs from the normal behavior of the musculoskeletal system observed during upright posture standing. In practice, imposing such closed-loop dynamics may at least result in a discomfortable standing bothering the patient. As emphasized previously, the main idea underlying the proposed control strategy is preserving the CoP and CoP velocity in a stable zone while their trajectories do not move towards the origin. In this manner, the balance dynamics can nearly imitate the balance dynamics of a healthy subject. Consequently, it can expect that utilizing such a control strategy not only can preserve the balance but also results in a comfortable standing of the patients.

\section{Comparison to the Similar Works}

As mentioned previously, controlling the CoP signal has not addressed directly in previous research works. Therefore, quantitative comparison to the similar presented works is cumbersome. However, the qualitative comparison seems rational. According to the results, the CoP dynamics controlled in a manner that it behaves in compliance with the CoP dynamics elicited in healthy subjects. However, using posture control strategies designed based on controlling the angle positions, as was done in the previous works, such dynamics conformity cannot be guaranteed. In fact, according to the achieved results, not only the joint angles were confined to the anatomical range of motion, but also the CoP dynamics has controlled directly.

\section{Conclusion}

In this work, a novel cooperative control strategy utilized for controlling the upright standing in paraplegia using FES. The cooperative control strategy designed based on a decision mechanism determined when the SMC or the adaptive controller has to be activated or deactivated. The results of the extended simulation studies on a musculoskeletal model, as a virtual patient, proves the acceptable and promising performance of the proposed strategy. Overall, it can conclude that the proposed cooperative control strategy is preferable in comparison to the single SMC and single adaptive controller.

Since the implementation of such experimental setup (implementation of electrical stimulators, proper hardware for bilateral communication with PC, related software, etc) was very time consuming, we would instead to present first the results of the comprehensive evaluation of the proposed control strategy on a musculoskeletal model. The next step of this research is providing a proper experimental setup for evaluation on the patients.

\section{Conflict of Interest Disclosures}

The authors declare that they have no conflict of interests.

\section{Ethical Statement}

Not applicable.

\section{Acknowledgments}

This work was supported by Research Center of Biomedical Department, Islamic Azad University, Mashhad, Iran.

\section{References}

1. Schauer T, Freeman C. Advances in functional electrical stimulation modelling and control. Med Eng Phys. 2016;38(11):1157-8.doi:10.1016/j.medengphy.2016.09.004.

2. Melo PL, Silva MT, Martins JM, Newman DJ. Technical developments of functional electrical stimulation to correct drop foot: sensing, actuation and control strategies. Clin Biomech (Bristol, Avon). 2015;30(2):101-13. doi: 10.1016/j. clinbiomech.2014.11.007.

3. Behboodi A, Zahradka N, Alesi J, Wright H, Lee SCK. Use of a novel functional electrical stimulation gait training system in 2 adolescents with cerebral palsy: a case series exploring neurotherapeutic changes. Phys Ther. 2019;99(6):739-47. doi: 10.1093/ptj/pzz040.

4. Nataraj R, Audu ML, Triolo RJ. Restoring standing capabilities with feedback control of functional neuromuscular stimulation following spinal cord injury. Med Eng Phys. 2017;42:13-25. doi: 10.1016/j.medengphy.2017.01.023.

5. Karimi MT. Evidence-based evaluation of physiological effects of standing and walking in individuals with spinal cord injury. Iran J Med Sci. 2011;36(4):242-53.

6. Braz GP, Russold MF, Fornusek C, Hamzaid NA, Smith RM, Davis GM. Cardiorespiratory and Muscle Metabolic Responses During Conventional Versus Motion Sensor-Assisted Strategies for Functional Electrical Stimulation Standing After Spinal Cord Injury. Artif Organs. 2015;39(10):855-62. doi: 10.1111/ aor.12619.

7. Rougier PR, Pérennou D. Upright standing after stroke: how loading-unloading mechanism participates to the postural stabilization. Hum Mov Sci. 2019;64:47-54. doi: 10.1016/j. humov.2019.01.004.

8. Masani K, Vette AH, Popovic MR. Controlling balance during quiet standing: proportional and derivative controller generates preceding motor command to body sway position observed in experiments. Gait Posture. 2006;23(2):164-72. doi: 10.1016/j.gaitpost.2005.01.006.

9. Vette AH, Masani K, Popovic MR. Implementation of a physiologically identified PD feedback controller for regulating the active ankle torque during quiet stance. IEEE Trans Neural Syst Rehabil Eng. 2007;15(2):235-43. doi: 10.1109/tnsre.2007.897016.

10. Rouhani H, Same M, Masani K, Li YQ, Popovic MR. PID controller design for FES applied to ankle muscles in neuroprosthesis for standing balance. Front Neurosci. 2017;11:347. doi: 10.3389/fnins.2017.00347.

11. Soetanto D, Kuo CY, Babic D. Stabilization of human standing posture using functional neuromuscular stimulation. J Biomech. 2001;34(12):1589-97. doi: 10.1016/s00219290(01)00144-0.

12. Huryn TP, Blouin JS, Croft EA, Koehle MS, Van der Loos HF. Experimental performance evaluation of human balance control models. IEEE Trans Neural Syst Rehabil Eng. 2014;22(6):1115-27. doi: 10.1109/tnsre.2014.2318351.

13. Hunt KJ, Munih M, de N Donaldson N. Feedback control of unsupported standing in paraplegia--part I: optimal control approach. IEEE Trans Rehabil Eng. 1997;5(4):331-40. doi: 10.1109/86.650287. 
14. Munih M, de N Donaldson N, Hunt KJ, Barr FM. Feedback control of unsupported standing in paraplegia--part II: experimental results. IEEE Trans Rehabil Eng. 1997;5(4):34152. doi: 10.1109/86.650288.

15. Hunt KJ, Gollee H, Jaime RP, de N Donaldson N. Design of feedback controllers for paraplegic standing. IEE ProceedingsControl Theory and Applications. 2001;148(2):97-108. doi: 10.1049/ip-cta:20010254.

16. Matjacić Z, Bajd T. Arm-free paraplegic standing--part I: control model synthesis and simulation. IEEE Trans Rehabil Eng. 1998;6(2):125-38. doi: 10.1109/86.681178.

17. Matjacić Z, Bajd T. Arm-free paraplegic standing--part II: experimental results. IEEE Trans Rehabil Eng. 1998;6(2):13950. doi: 10.1109/86.681179.

18. Mihelj M, Munih M. Unsupported standing with minimized ankle muscle fatigue. IEEETrans Biomed Eng. 2004;51 (8):133040. doi: 10.1109/tbme.2004.827560.

19. Audu ML, Odle BM, Triolo RJ. Control of standing balance at leaning postures with functional neuromuscular stimulation following spinal cord injury. Med Biol Eng Comput. 2018;56(2):317-30. doi: 10.1007/s11517-017-1687-x.

20. Kobravi HR, Erfanian A. Decentralized adaptive robust control based on sliding mode and nonlinear compensator for the control of ankle movement using functional electrical stimulation of agonist-antagonist muscles. J Neural Eng. 2009;6(4):046007. doi: 10.1088/1741-2560/6/4/046007.

21. Kobravi HR, Erfanian A. A decentralized adaptive fuzzy robust strategy for control of upright standing posture in paraplegia using functional electrical stimulation. Med Eng Phys. 2012;34(1):28-37. doi: 10.1016/j.medengphy.2011.06.013.

22. Loram ID, Lakie M. Human balancing of an inverted pendulum: position control by small, ballistic-like, throw and catch movements. J Physiol. 2002;540(Pt 3):1111-24. doi: 10.1113/jphysiol.2001.013077.

23. Jafari H, Nikolakopoulos G, Gustafsson T. Stabilization of an Inverted Pendulum via Human Brain Inspired Controller Design. IEEE-RAS International Conference on Humanoid Robots; 2019.

24. Wang Q, Sharma N, Johnson M, Gregory CM, Dixon WE. Adaptive Inverse optimal neuromuscular electrical stimulation. IEEE Trans Cybern. 2013;43(6):1710-8. doi: 10.1109/tsmcb.2012.2228259.

25. Balasubramaniam R. On the control of unstable objects: the dynamics of human stick balancing. Adv Exp Med Biol. 2013;782:149-68. doi: 10.1007/978-1-4614-5465-6_8.

26. Ruhbakhsh SS, Kobravi HR. Preserving balance during unsupported standing using synergistic combination of discrete sliding predictive and adaptive controllers. Proceedings of IEEE International congress on Technology, Communication and Knowledge (ICTCK), Iran. 2015:218-25

27. Li Y, Levine WS. An Optimal Control Model for Human Postural Regulation. American Control Conference; 2009. p. 4705-10.

28. Winter DA, Patla AE, Prince F, Ishac M, Gielo-Perczak K. Stiffness control of balance in quiet standing. J Neurophysiol. 1998;80(3):1211-21. doi: 10.1152/jn.1998.80.3.1211.

29. Ferrarin M, Palazzo F, Riener R, Quintern J. Model-based control of FES-induced single joint movements. IEEE Trans Neural Syst Rehabil Eng. 2001;9(3):245-57. doi: 10.1109/7333.948452.

30. Popovic M, Pappas IP, Nakazawa K, Keller T, Morari M, Dietz V. Stability criterion for controlling standing in able-bodied subjects. J Biomech. 2000;33(11):1359-68. doi: 10.1016/ s0021-9290(00)00123-8.

31. Cho GH, Hwangbo G, Shin HS. The effects of virtual realitybased balance training on balance of the elderly. J Phys Ther Sci. 2014;26(4):615-7. doi: 10.1589/jpts.26.615.

32. Mobayen S, Tchier F. Nonsingular fast terminal sliding-mode stabilizer for a class of uncertain nonlinear systems based on disturbance observer. Sci Iran. 2017;24(3):1410-8. doi: 10.24200/sci.2017.4123.

33. Parsapour M, RayatDoost S, Taghirad HD. A 3D sliding mode control approach for position based visual servoing system. Sci Iran. 2015;22(3):844-53.

34. Ginoya D, Shendge PD, Phadke SB. Sliding mode control for mismatched uncertain systems using an extended disturbance observer. IEEE Trans Ind Electron. 2013;61(4):1983-92. doi: 10.1109/tie.2013.2271597.

35. Utkin VI. Variable structure systems with sliding modes. IEEE Trans Automat Contr. 1977;22(2):212-22. doi: 10.1109/ tac.1977.1101446.

36. Yin C, Dadras S, Zhong SM, Chen YQ. Control of a novel class of fractional-order chaotic systems via adaptive sliding mode control approach. Appl Math Model. 2013;37(4):2469-83. doi: 10.1016/j.apm.2012.06.002.

37. Xiao L, Su H, Chu J. Sliding mode prediction based control algorithm for discrete-time non-linear uncertain coupled systems. Int J Control. 2007;80(10):1616-25. doi: 10.1080/00207170701447122.

38. Xiao L, Duan S, Shen T. Time-Varying Sliding Mode Control for Discrete-Time Nonlinear Uncertain Coupled Systems via Sliding Mode Prediction. Applied Mechanics and Mechanical Engineering. 2010;29(1):2164-9.

39. Slotine JJ, Li W. Applied Nonlinear Control. Prentice Hall; 1991.

40. Fu SHS, Cheng CC. Direct Adaptive Control Designs for Nonlinear Discrete-Time Systems with Matched Uncertainties. Proceedings of the 2005 IEEE International Conference on Mechatronics; 10-12 July 2005; Taipei, Taiwan. p. 881-6. doi: 10.1109/icmech.2005.1529378.

41. Rugh WJ. Linear System Theory. Upper Saddle River, NJ, USA: Practice Hall; 1996.

42. Fu SHS, Cheng CC. Adaptive Stabilization for Normal Nonlinear Discrete- Time Uncertain Systems. In: Proceedings of the 2004 5th Asian Control Conference; 2004; Kaohsiung, Taiwan. p. 2032-8.

43. Tortolero X, Masani K, Popovic MR. Step prediction during perturbed standing using center of pressure measurements. Sensors (Basel). 2007;7(4):459-72.

44. Callahan DM, Umberger BR, Kent-Braun JA. A computational model of torque generation: neural, contractile, metabolic and musculoskeletal components. PLoS One. 2013;8(2):e56013. doi: 10.1371/journal.pone.0056013. 